\title{
The neural basis of Tetris gameplay: implicating the role of visuospatial processing
}

\author{
Thomas Agren $^{1}$ - Johanna M. Hoppe ${ }^{1}$ (D) $\cdot$ Laura Singh $^{1} \cdot$ Emily A. Holmes $^{1,2} \cdot$ Jörgen Rosén $^{1}$
}

Accepted: 5 July 2021

(C) The Author(s) 2021

\begin{abstract}
Tetris is not only a widely used entertaining computer game, but has been used as a component in emerging psychological interventions targeting dysfunctional mental imagery, e.g., intrusive memories and imagery-based cravings. However, little is known about the neurobiological mechanisms underlying these interventions. Tetris gameplay has been hypothesized to disrupt dysfunctional mental imagery (e.g., imagery-based intrusive memories of adverse events) and cravings (e.g., substance use) by taxing visuospatial working memory. In line with this, the present study aimed to characterize brain areas involved in the visuospatial aspects of Tetris gameplay, by controlling for motor activity (button presses) and using gameplay instructions emphasizing mental rotation. Participants $(N=28)$ received mental rotation instructions and thereafter either played Tetris, or only pressed buttons as if playing Tetris (motor activity), while undergoing functional magnetic resonance imaging. Tetris gameplay (when using mental rotation instructions and controlling for motor activity) robustly activated brain areas located in the ventral and dorsal stream, with maximum peak activation in the inferior and mid temporal gyrus. To the best of our knowledge, this is the first study to characterize brain areas specifically associated with the visuospatial aspects of Tetris gameplay, by controlling for motor activity and when using mental rotation instructions. Results demonstrate that engaging in Tetris gameplay recruits an extensive brain circuitry previously tied to visuospatial processing. Thus, findings are consistent with the use of Tetris as an imagery-competing task as one of several components of emerging interventions targeting dysfunctional mental imagery.
\end{abstract}

Keywords Tetris $\cdot$ Intrusive memories $\cdot$ Visuospatial task $\cdot$ Functional magnetic resonance imaging $\cdot$ Mental imagery

\section{Introduction}

Tetris is a computer game where geometric blocks of different shapes and colors fall from the top of the screen in a random sequence one at a time. The task of the player is to rotate and place these blocks at the bottom of the screen. Fitting blocks together to complete horizontal lines is rewarded with points and extended game time. The immersive nature of Tetris gameplay, often capturing the player's full attention towards the game, and its imagery-competing (e.g. visuospatial) aspects has recently been harnessed in the development of novel psychological interventions (Singh et al., 2020; Skorka-

Thomas Agren

thomas.agren@psyk.uu.se

1 Department of Psychology, Uppsala University, Box 1225, SE-751 42 Uppsala, Sweden

2 Department of Clinical Neuroscience, Karolinska Institutet, Stockholm, Sweden
Brown et al., 2014). In particular, Tetris is being used as one component within psychological interventions to reduce dysfunctional mental imagery. A novel behavioral intervention, involving Tetris gameplay together with specific instructions to engage in mental rotation (i.e., as part of several intervention components), has shown promise to both prevent the development of intrusive memories (Horsch et al., 2017; Iyadurai et al., 2018; Kanstrup et al., 2021a) and reduce the frequency of long-standing intrusive memories after trauma (Kanstrup et al., 2021b; Kessler et al., 2018). Another procedure involving Tetris gameplay has been shown to reduce the strength, frequency, and vividness of dysfunctional cravings (e.g., alcohol, food, nicotine; Skorka-Brown et al., 2014; Skorka-Brown et al., 2015). Tetris has also been used within a procedure to reduce the emotional intensity and vividness of mental imagery during recall of emotional memories (Engelhard et al., 2010). Nevertheless, what makes Tetris a successful tool in psychological interventions is still incompletely understood, including what Tetris gameplay entails at the neural level. 
Mental imagery refers to sensory experiences in the absence of external sensory input (Holmes \& Agren, 2020). A prominent feature of mental imagery is that it provokes similar emotional (Holmes \& Mathews, 2005), physiological and neural responses (Hoppe et al., 2021; Pearson, 2019) "as if" the imagined event was encountered in real life (e.g., imaging vs. seeing a spider). The impact of mental imagery on emotion is indisputably manifested in posttraumatic stress disorder in which distressing, intrusive mental imagery of past trauma constitutes a core symptom (American Psychiatric Association, 2013). Evidence indicates that visual mental imagery hinges on visuospatial working memory (Baddeley \& Andrade, 2000). For instance, taxing working memory resources with a concurrent visuospatial task reduces the vividness (liveliness and clarity) and emotional impact of mental imagery (Bywaters et al., 2004; Kavanagh et al., 2001). Tetris is hypothesized to act as a cognitive task that competes with mental imagery for working memory resources when, for example, an emotional memory is held in mind (e.g., Engelhard et al., 2010; Lau-Zhu et al., 2017; Skorka-Brown et al., 2014). That is, Tetris is hypothesised to place competition for limited working memory and can therefore act as an imagerycompeting interference task to reduce the frequency or intensity of dysfunctional mental imagery.

The behavioral intervention that targets intrusive memories after trauma is based on the hypothesis that Tetris gameplay can interfere with the initial consolidation of the visuospatial mental imagery components of recent trauma memories (Deeprose et al., 2012) or the reconsolidation of already established, older trauma memories (James et al., 2015), by taxing visuospatial working memory specifically (Lau-Zhu et al., 2017). It is hypothesized that the active use of mental rotation during Tetris gameplay (i.e., visualizing the upcoming blocks in the game and rotating them in the mind's eye to work out where they best fit) promotes the effects of the intervention by increasing mental imagery and visuospatial aspects of the way the game is played. Specific instructions emphasizing mental rotation is therefore thought to be one important component in protocols using this intervention (Iyadurai et al., 2018; Kanstrup et al., 2021a, b; Kessler et al., 2018). The use of Tetris as a visuospatial intervention to combat cravings is rooted in the elaborated intrusion (EI) theory (Kavanagh et al., 2005). EI considers cravings to be cognitive-emotional states involving embodied sensory imagery, and that a visuospatial interference task can compete with the resources used for the intrusive imagery and thus neutralize the initiated emotions of cravings (Skorka-Brown et al., 2014, 2015). Although interventions for intrusive memories and cravings emphasize that the usefulness of Tetris gameplay hinges on its capacity to compete with mental imagery and load visuospatial working memory specifically (but see Engelhard et al., 2010), little is known about the neurobiological mechanisms underlying these intervention procedures, and the neural basis of Tetris gameplay, such as its proposed visuospatial aspects, are not fully understood.

Tetris gameplay has previously been used in neuroimaging studies to investigate brain activity in emotion regulatory neural circuits (amygdala and occipital cortex; Price et al., 2013), to examine the impact of repeated practice of a visuospatial/ motor task on overall brain activity (Haier et al., 1992; Rietschel et al., 2012), and to assess the plasticity of cortical grey matter (Haier et al., 2009). Also, a neuroimaging study on posttraumatic stress disorder (PTSD) showed that daily Tetris gameplay (3 months), in conjunction with psychological treatment, increased hippocampal volume, which was associated with PTSD symptom reduction (Butler et al., 2020). However, there is a need for further neuroimaging studies pinpointing the visuospatial aspects of Tetris gameplay. Brain areas involved in the visuospatial aspects of Tetris gameplay could be examined more specifically by controlling for the motor activity elicited by button presses during gameplay, and by using instructions emphasizing mental rotation during gameplay to boost mental imagery and visuospatial processing. Identifying the neural underpinnings of Tetris gameplay may help contribute to a mechanistic understanding of visuospatial interference tasks, which, in turn, could inform ways to optimize existing interventions and promote the development of other visuospatial interventions with therapeutic effects (Holmes et al., 2018; Singh et al., 2020).

\section{Aim of the Study}

The present study aimed to characterize brain areas involved in visuospatial aspects of Tetris gameplay, by controlling for motor activity (button presses), and using gameplay instructions emphasizing mental rotation during gameplay (in line with how it is used in emerging intervention protocols). To this end, we used functional magnetic resonance imaging (fMRI) and a subtraction design to identify brain areas activated during Tetris gameplay while controlling for motor activity. To mirror a clinical implementation of Tetris gameplay, we use the same instructions on mental rotation as those given within an intervention procedure targeting the reduction of intrusive memories after trauma (Iyadurai et al., 2018; Kanstrup et al., 2021a, b; Kessler et al., 2018;).

\section{Methods and Materials}

\section{Participants}

Thirty participants were recruited through advertisements on social media and billboards. Exclusion criteria consisted of current psychiatric disorder (other than spider phobia), substance abuse, neurological disease, receiving psychological treatment or psychotropic medication within six months, and 
contraindications for magnetic resonance imaging. Data was collected as part of an unrelated experiment where one of the inclusion criteria was fear of spiders (Hoppe et al., 2021). Twenty-eight participants (age: $\mathrm{M}=23.8, \mathrm{SD}=5.8$ years, 7 men and 21 women) were included in analyses. All participants, except for one, were right-handed. One participant was excluded from analyses as the participant did not follow the instructions, and another participant was excluded because of missing data. The study was approved by the national Research Ethics Committee. All participants provided written and informed consent. Participants were paid an amount corresponding to $\$ 50$ for their participation. The data is available in the Open Science Framework [https://osf.io/qj8xc/].

\section{Primary Outcome Measure}

The primary outcome measure was brain activity, defined as blood oxygen level dependent (BOLD) signal assessed with fMRI, during Tetris gameplay while controlling for motor activity.

\section{Materials}

For use in the fMRI scanner, an in-house Tetris app was programmed using the Unity graphics engine (version 5.2.3, Unity Technologies, San Francisco, CA). Two hand controls using pistol grip (Nordic NeuroLab, Bergen, Norway), with two buttons each (thumb and index finger), were used for gameplay in the scanner.

\section{Procedure}

Before entering the scanner, participants were instructed on how to play Tetris using active mental rotation (i.e., to visualize in their mind's eye how to rotate/move the Tetris blocks to fit them into horizontal lines - planning ahead for the next block to appear on the screen during gameplay; see Supplementary materials for protocol). Left and right index fingers were used to steer the blocks left and right. The left thumb was used to rotate blocks, and the right thumb to move the block down faster. Participants practiced the controls outside of the scanner, using corresponding fingers on a laptop until they were confident about the layout of the controls and how to play Tetris with active mental rotation.

The scanner procedure was $7 \mathrm{~min}$ long. It consisted of four cycles of Tetris gameplay $(80 \mathrm{~s}, 60 \mathrm{~s}, 60 \mathrm{~s}, 60 \mathrm{~s})$ interleaved with four cycles of motor activity (20 s) directly followed by a resting period ( $20 \mathrm{~s}$; see Supplementary Fig. S1). In the motor activity condition, participants were instructed to keep pressing the buttons of the hand controls as if still playing Tetris. The motor activity condition always followed Tetris gameplay to keep button pressing as similar as possible between these conditions (participants kept pressing buttons as during immediate preceding gameplay). Instructions during the rest condition (baseline) were to relax and wait for the game to start again. Participants were instructed to keep their eyes open during all conditions (Tetris gameplay, motor activity, rest).

\section{Brain Imaging}

Data were acquired using a $3 \mathrm{~T}$ whole-body MR scanner (Philips Achieva 3.0 T TX, Philips Medical Systems, Best, The Netherlands) with a 32-channel head coil. First, an anatomical $\mathrm{T}_{1}$-weighted reference image (voxel size $0.938 \times$ $0.938 \times 1$; matrix $256 \times 256$; 220 slices) was collected. During the experimental session, blood oxygen level dependent (BOLD) imaging was performed using a single-shot EPI sequence with whole-brain coverage $(\mathrm{TE} / \mathrm{TR}=35 / 3000 \mathrm{~ms}$; flip angle $90^{\circ}$; acquired voxel size $1.5 \times 1.5 \times 3.0$, matrix $160 \times 160,45$ slices with no gap in ascending order). BOLD images were normalized to the Montreal Neurological Institute (MNI) standard space, slice time corrected, and spatially smoothed using an $8 \mathrm{~mm}$ FWHM kernel (voxel size $2 \times$ $2 \times 2$ ). Preprocessing and all analyses of fMRI-data were performed using SPM12 (Wellcome Department of Cognitive Neurology, University College, London (http://www.fil. ion. ucl.ac.uk).

\section{Data Analysis}

For first-level analyses, two experimental regressors were created, one for Tetris gameplay and one for motor activity (i.e., button presses). Rest periods were left to form the baseline. In line with the aim of the study, the primary second-level analysis was the contrast Tetris gameplay relative to motor activity, which assessed brain activations during Tetris gameplay while controlling for motor activity. Additionally, a secondlevel analysis was performed for Tetris gameplay relative to baseline (rest) to reveal brain activations during Tetris gameplay with accompanying motor activity. Finally, a second-level analysis was conducted for motor activity relative to baseline to show brain activation during motor activity only. Analyses used familywise error correction $p_{\mathrm{FWE}}<0.05$ over the whole brain. The Statistical Parametric Mapping (SPM) anatomy toolbox (Eickhoff et al., 2005) was used to extract activated clusters and label areas of local maximum. The xjView toolbox (https://www.alivelearn.net/xjview) for SPM and the automated anatomical labeling (aal) atlas (Tzourio-Mazoyer et al., 2002) was used to identify and label brain areas located within each cluster.

\section{Results}

Results demonstrate that Tetris gameplay, after being given instructions to engage in mental rotation, and when 
controlling for the motor activity of pressing buttons, activated brain areas in occipital, temporal, parietal, and premotor areas. The global peaks were situated bilaterally in the posterior parts of the inferior temporal gyrus (inferotemporal cortex) and the middle temporal gyrus. Local maxima were found bilaterally in the fusiform gyrus, middle and inferior occipital gyrus, thalamus, superior frontal gyrus, angular gyrus, cerebellum, and the midcingulate cortex. Lateral local maxima were found in several areas, among them the hippocampus. Complete results are presented in Table 1 and further illustrated in Fig. 1. See Supplementary Table S1 for local maxima for both Tetris gameplay relative to motor activity, and motor activity relative to Tetris gameplay, and Supplementary Table S2 for a complete list of brain areas laying within each cluster. Brain activations during Tetris gameplay relative to baseline are illustrated in Fig. 2, reflecting brain activations associated with both the visuospatial (e.g., mental rotation) and motor aspects (button presses to move the blocks) of Tetris gameplay. See Supplementary Table S3 for results regarding Tetris gameplay relative to baseline and Supplementary Table S4 for a complete list of brain areas within each cluster for this contrast. Corresponding results for the contrast motor activity relative to baseline are presented in Supplementary Table S5 (areas of local maximum) and Supplementary Table S6 (brain areas within each cluster). The mean frequency of button presses (i.e button presses per second) during Tetris gameplay $(M=1.76, S D=0.85)$ and the motor activity condition (i.e., button presses only; $M=1.58, S D=0.74$ ) did not differ significantly, $t(27)=1.44, p=0.16, \mathrm{~d}=0.44$.

\section{Discussion}

The present study aimed to characterize brain areas specifically associated with the visuospatial aspects of Tetris gameplay, by controlling for motor activity (button presses), and using instructions emphasizing mental rotation during gameplay. Results demonstrated that visuospatial processing during Tetris gameplay was associated with robust activation of brain areas across the ventral and dorsal visual stream, i.e., brain areas previously tied to processing of visuospatial information (Shetht \& Young, 2016). For instance, the global peak activations during Tetris gameplay were found bilaterally in the posterior parts of the inferior temporal gyrus (inferotemporal cortex) and the middle temporal gyrus. The inferotemporal cortex is at the apex of the ventral visual stream, providing viewinvariant identification of objects for use in higher cortical functions (Conway, 2018). This function is consistent with what is performed during the experimental task, where blocks on the screen must be transformed into view-invariant representations for which internal, i.e., mental rotations can be performed. Object recognition has also been related to activity in the neighboring fusiform gyrus (Weiner et al., 2018), which was robustly activated in the current study. Tetris gameplay was also associated with increased activity in premotor areas, even when controlling for motor activity, in line with an engagement in motor planning and decision-making during Tetris gameplay (Hardwick et al., 2013). These activations, together with widespread activation of the occipital cortex, show that Tetris gameplay activates areas tied to visuospatial processing. Thus, findings are consistent with the hypothesis behind emerging interventions targeting dysfunctional mental imagery, that is, that it may be possible to use Tetris gameplay to tax visuospatial processing resources, i.e. compete with mental imagery.

Current results are consistent with findings from previous neuroimaging studies examining other processes related with Tetris gameplay (e.g., the effects of daily practice, task difficulty of Tetris gameplay). In these studies, Tetris gameplay was also associated with activations in the occipital and parietal lobes (Haier et al., 2009; Price et al., 2013) as well as the premotor areas (Haier et al., 2009). Notably, we did find a local maxima (Supplementary Table S1) and many activated voxels within the hippocampus (Supplementary Table S2), an area in which volume has been observed to increase with daily Tetris gameplay practice (3 months; Butler et al., 2020). In the current study, the global peak during Tetris gameplay, controlling for motor activity, was in the middle temporal gyrus (Table 1). Interestingly, experimental studies examining the neural mechanisms involved in the formation of intrusive memories suggest that activation of the middle temporal gyrus may distinguish whether a specific stimulus will become an intrusive memory or not (Bourne et al., 2013; Clark et al., 2016), albeit that the activation in middle temporal gyrus in the present study was further back (BA 19). Thus, this brain area may offer a potential link between visuospatial processing and the formation of memories that become intrusive visual imagery. Future studies should extend our findings by investigating the brain mechanisms underlying Tetris gameplay when it is used as an interference task to target mental imagery-based intrusive memories, i.e., during trauma recall. Relating the reduction in frequency of intrusive memories to neural processing during Tetris gameplay could help uncover underlying mechanisms of how Tetris gameplay can prevent the formation and expression of intrusive memories.

The current study focused on a subtraction design to isolate the neural basis of the visuospatial aspects of Tetris (rather than the motor aspect of button pressing during gameplay), because visuospatial (i.e. imagery-competing) processing has been hypothesized to be one of several important components for the clinical effects of Tetris gameplay when used as an interference task in psychological interventions (Lau-Zhu et al., 2017; Skorka-Brown et al., 2014, 2015). However, the motor aspects of Tetris gameplay (that is, the act of pressing buttons itself) may contribute to the beneficial effects, for instance, by increasing the load on working memory. Indeed, the 
Table 1. Brain areas activated during Tetris gameplay*.

\begin{tabular}{|c|c|c|c|c|c|c|}
\hline \multirow[b]{2}{*}{ Cluster $^{\mathrm{a}}$} & \multirow[b]{2}{*}{ Area of local maximum ${ }^{b}$} & \multirow[b]{2}{*}{$t$} & \multicolumn{3}{|c|}{ MNI** } & \multirow[b]{2}{*}{ Voxels (cluster) } \\
\hline & & & $\mathrm{x}$ & $\mathrm{y}$ & $\mathrm{z}$ & \\
\hline \multirow[t]{10}{*}{1} & $\begin{array}{l}\text { R Middle Temporal Gyrus } \\
\text { R Inferior Temporal Gyrus }\end{array}$ & $\begin{array}{l}21.71 \\
20.38\end{array}$ & $\begin{array}{l}46 \\
44\end{array}$ & $\begin{array}{l}-72 \\
-62\end{array}$ & $\begin{array}{l}-2 \\
-8\end{array}$ & \multirow[t]{10}{*}{23,107} \\
\hline & L Inferior Parietal Lobule & 18.99 & -26 & -54 & 52 & \\
\hline & L Inferior Occipital Gyrus & 18.11 & -42 & -66 & -4 & \\
\hline & L Middle Occipital Gyrus & 17.12 & -42 & -78 & 2 & \\
\hline & R Middle Occipital Gyrus & 16.09 & 36 & -84 & 2 & \\
\hline & R Fusiform Gyrus & 16.03 & 26 & -70 & -14 & \\
\hline & R Inferior Occipital Gyrus & 15.96 & 34 & -82 & -2 & \\
\hline & R Middle Occipital Gyrus & 15.81 & 30 & -80 & 26 & \\
\hline & L Fusiform Gyrus & 15.43 & -28 & -60 & -10 & \\
\hline & L Fusiform Gyrus & 15.38 & -26 & -70 & -6 & \\
\hline \multirow[t]{10}{*}{2} & R Thalamus & 13.18 & 20 & -28 & 12 & \multirow[t]{10}{*}{1639} \\
\hline & n.a. & 11.80 & -6 & -30 & -4 & \\
\hline & n.a. & 11.68 & 6 & -32 & -4 & \\
\hline & n.a. & 9.38 & 2 & -24 & -20 & \\
\hline & L Thalamus & 9.06 & -16 & -28 & 14 & \\
\hline & R Thalamus & 7.99 & 10 & -20 & 4 & \\
\hline & n.a. & 7.41 & -4 & -32 & -20 & \\
\hline & n.a. & 7.08 & 12 & -18 & -6 & \\
\hline & L Thalamus & 6.31 & -8 & -16 & 10 & \\
\hline & L Thalamus & 6.09 & -8 & -14 & 14 & \\
\hline \multirow[t]{6}{*}{3} & R Superior Frontal Gyrus & 9.86 & 26 & -8 & 66 & \multirow[t]{6}{*}{610} \\
\hline & R Superior Frontal Gyrus & 9.78 & 32 & -6 & 62 & \\
\hline & R Precentral Gyrus & 9.18 & 30 & -4 & 48 & \\
\hline & n.a. & 8.92 & 34 & -8 & 44 & \\
\hline & R Superior Frontal Gyrus & 7.23 & 20 & -8 & 72 & \\
\hline & R Superior Frontal Gyrus & 6.83 & 28 & 4 & 64 & \\
\hline 4 & L Superior Frontal Gyrus & 10.18 & -20 & -6 & 58 & 383 \\
\hline 5 & R Inferior Frontal Gyrus & 8.64 & 48 & 6 & 26 & 179 \\
\hline \multirow[t]{2}{*}{6} & n.a. & 8.34 & -6 & 24 & 6 & \multirow[t]{2}{*}{63} \\
\hline & n.a. & 6.59 & 2 & 22 & 4 & \\
\hline 7 & R Mid Cingualte Cortex & 6.96 & 14 & -30 & 42 & 21 \\
\hline
\end{tabular}

Note. *Visuospatial processing during Tetris gameplay was assessed by controlling for motor activity (button presses) and using instructions emphasizing mental rotation. ${ }^{a}$ Results are presented by clusters of activation, starting with the largest. ${ }^{b}$ Area of local maximum identifies the locations of peak voxels (strongest BOLD-signal) within each cluster. $\mathrm{L}=$ left. $\mathrm{R}=$ right hemisphere. $\mathrm{n} . \mathrm{a}$. = brain area not defined in anatomical brain atlas. **Coordinates $(\mathrm{x}, \mathrm{y}, \mathrm{z})$ refer to the Montreal Neurological Institute template. Results are familywise errorcorrected $p_{\text {FWE }}<.0 .05$

combination of both visuospatial and motor aspects may be required for Tetris to be effective as an interference task. Furthermore, mental imagery is not restricted to sensory experiences, but can also involve simulating motion and motor behavior. Just as producing mental imagery of an object, and seeing the same object (e.g., a spider), recruit similar neurocircuitry and have a similar impact on emotion (Hoppe et al., 2021; Pearson, 2019), so does imagining and executing a motor action (e.g., running; Munzert et al., 2009). It has been suggested that interference tasks need to be in the same modality as the targeted imagery to be effective (e.g., visuospatial task to target visual mental imagery; Baddeley \& Andrade, 2000). Hence, the motor aspects of Tetris could potentially promote the impact on motor imagery (e.g., running away from a perpetrator). Lang has long proposed that mental imagery involves preparedness for action (Lang, 1979), and planning actions and making movements are both components of Tetris gameplay. Future studies should explore the intricate relation between visuospatial and motor aspects of Tetris gameplay and their impact on emotional mental imagery. 
Fig. 1 (A) Tetris gameplay after receiving mental rotation instructions, and controlling for motor activity (button presses), recruits brain areas involved in visuospatial processing, including areas in the occipital, temporal, and frontal lobes (premotor areas see also Table 1). (B) Composite image depicts brain activation of Tetris gameplay, controlling for motor activity (in red: same contrast as in A) in relation to brain activations elicited by motor activity only, showing both contrasts overlaid on the same brain template (axial, sagittal, and coronal view). All images display whole-brain familywise errorcorrected results, $p_{\text {FWE }}<0.05$

A Brain areas activated during Tetris gameplay, controlling for motor activity
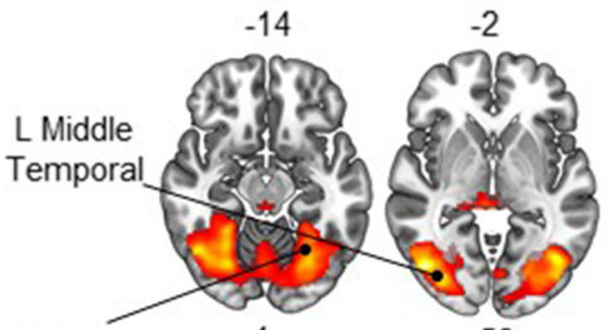

58

R Fusiform

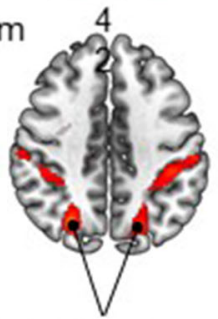

L/R Superior

Occipital

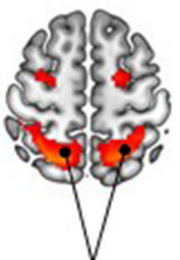

L/R Inferior

Occipital

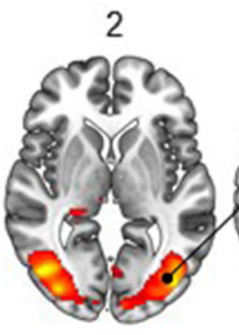

64

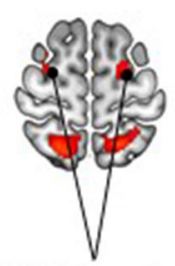

L/R Superior

Frontal
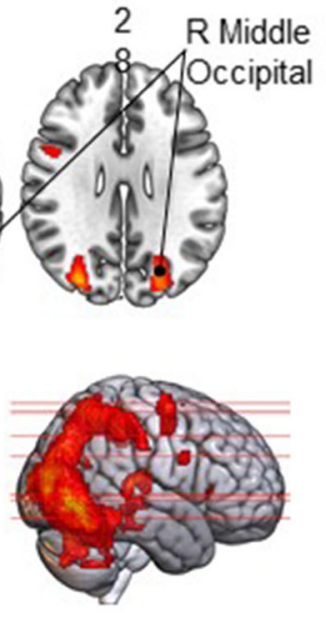

กั

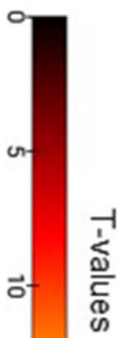

B Composite image of brain areas activated during Tetris gameplay, controlling for motor activity, and activation during motor activity only

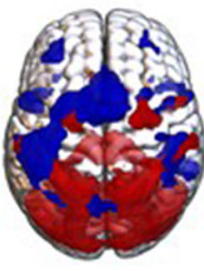

Axial view

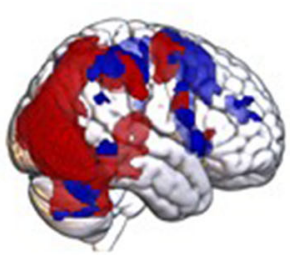

Sagittal view

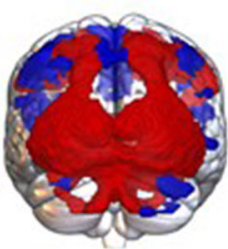

Coronal view
- Tetris gameplay, controlling for motor activity

- Motor activity relative to baseline

\section{Limitations}

Some limitations should be taken into consideration when interpreting the current results. The experimental procedure included longer durations of Tetris gameplay ( $80-60 \mathrm{~s} / \mathrm{block}$ ) compared to the condition of assessing motor activity only (button presses: $20 \mathrm{~s} / \mathrm{block}$ ). Thus, less data (i.e., a smaller number of brain volumes) was collected for motor activity as compared with Tetris gameplay. However, an additional analysis, matching the durations of Tetris gameplay and motor activity to $20 \mathrm{~s}$ (i.e., the last $20 \mathrm{~s}$ of each period of Tetris gameplay was used in the analysis), produced similar results (see Supplementary Table S7 for a list of local maxima and Supplementary Table S8 for a complete list of brain areas laying within each cluster).

It should also be noted that we could not assess to what extent participants engaged in mental rotation during gameplay, despite instructions to do so. Future studies should
Fig. 2 Brain activations during Tetris gameplay together with motor activity of pressing buttons. The figure displays the contrast of Tetris gameplay (with button presses) relative to baseline (rest), illustrating both the visuospatial (e.g., mental rotation) and motor aspects (button presses) of Tetris gameplay together

\section{A Brain areas activated during Tetris gameplay including motor activity}
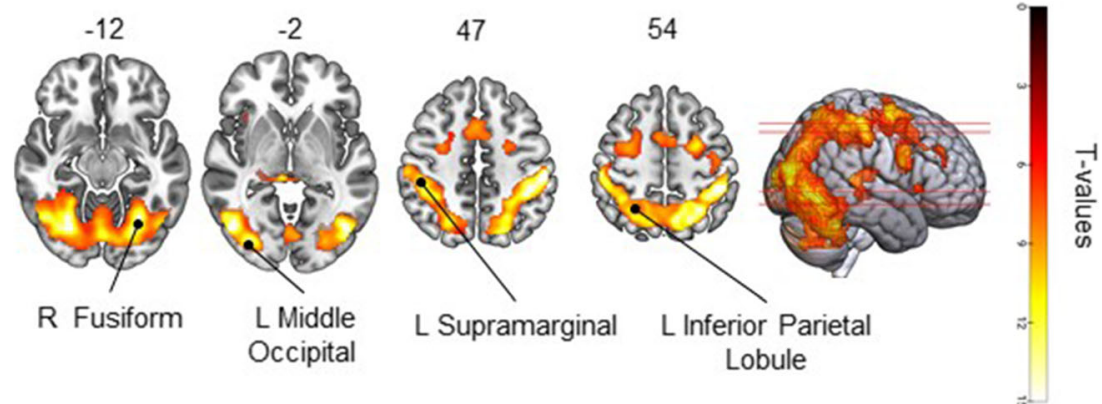
explore to what extent mental rotation instructions impact activation of visuospatial processing brain areas, as well as impact associated clinically relevant effects (e.g., the reduction in frequency of intrusive memories). Lastly, the majority of the sample consisted of female participants. Future studies should include a more balanced sample to detect potential gender differences in neural activity associated with visuospatial processing during Tetris gameplay.

\section{Conclusions}

To the best of our knowledge, this is the first study to characterize brain activity specifically associated with visuospatial processing during Tetris gameplay. Results demonstrate that Tetris gameplay, when controlling for the motor activity of pressing buttons, and after being given instructions to engage in mental rotation, recruits an extensive brain circuitry previously associated with neural processing of visuospatial information. Findings are consistent with the use of Tetris as an imagery-competing task as one of several components of emerging interventions targeting dysfunctional mental imagery. Understanding the underlying neural processes of Tetris gameplay may help to optimize the effectiveness of such interventions, promote the development of novel interventions, as well as contribute to a mechanistic understanding of visuospatial interference tasks.

Supplementary Information The online version contains supplementary material available at https://doi.org/10.1007/s12144-021-02081-z.

Funding Open access funding provided by Uppsala University. TA was supported by the Swedish Research Council (2014-01335) and the Bank of Sweden Tercentenary Foundation (P18-0487:1). EAH received grant support from the Swedish Research Council (2017-00957 and 202000873), the Oak Foundation (OCAY-18-442), AFA Insurance (200342), and the Lupina Foundation. LS was funded by the Swiss National Science Foundation (P2BEP1 184378) and a Thunberg Fellowship by the Swedish Collegium for Advanced Study.

Open Access This article is licensed under a Creative Commons Attribution 4.0 International License, which permits use, sharing, adaptation, distribution and reproduction in any medium or format, as long as you give appropriate credit to the original author(s) and the source, provide a link to the Creative Commons licence, and indicate if changes were made. The images or other third party material in this article are included in the article's Creative Commons licence, unless indicated otherwise in a credit line to the material. If material is not included in the article's Creative Commons licence and your intended use is not permitted by statutory regulation or exceeds the permitted use, you will need to obtain permission directly from the copyright holder. To view a copy of this licence, visit http://creativecommons.org/licenses/by/4.0/.

\section{References}

American Psychiatric Association. (2013). Diagnostic and statistical manual of mental disorders (DSM-5®). https://doi.org/10.1176/ appi.books.9780890425596
Baddeley, A. D., \& Andrade, J. (2000). Working memory and the vividness of imagery. Journal of experimental psychology General, 129(1), 126-145. https://doi.org/10.1037/0096-3445.129.1.126.

Bourne, C., MacKay, C. E., \& Holmes, E. A. (2013). The neural basis of flashback formation: The impact of viewing trauma. Psychological Medicine, 43(7), 1521-1532. https://doi.org/10.1017/ S0033291712002358.

Butler, O., Herr, K., Willmund, G., Gallinat, J., Kuhn, S., \& Zimmermann, P. (2020). Trauma, treatment and Tetris: Video gaming increases hippocampal volume in male patients with combatrelated posttraumatic stress disorder. Journal of Psychiatry and Neuroscience, 45(4), 279-287. https://doi.org/10.1503/jpn.190027.

Bywaters, M., Andrade, J., \& Turpin, G. (2004). Determinants of the vividness of visual imagery: The effects of delayed recall, stimulus affect and individual differences. Memory, 12(4), 479-488. https:// doi.org/10.1080/09658210444000160.

Clark, I. A., Holmes, E. A., Woolrich, M. W., \& Mackay, C. E. (2016). Intrusive memories to traumatic footage: The neural basis of their encoding and involuntary recall. Psychological Medicine, 46(3), 505-518. https://doi.org/10.1017/S0033291715002007.

Conway, B. R. (2018). The organization and operation of inferior temporal cortex. Annual Review of Vision Science, 4(1), 381-402. https://doi.org/10.1146/annurev-vision-091517-034202.

Deeprose, C., Zhang, S., Dejong, H., Dalgleish, T., \& Holmes, E. A. (2012). Imagery in the aftermath of viewing a traumatic film: Using cognitive tasks to modulate the development of involuntary memory. Journal of Behavior Therapy and Experimental Psychiatry, 43(2), 758-764. https://doi.org/10.1016/j.jbtep.2011. 10.008 .

Eickhoff, S. B., Stephan, K. E., Mohlberg, H., Grefkes, C., Fink, G. R., Amunts, K., \& Zilles, K. (2005). A new SPM toolbox for combining probabilistic cytoarchitectonic maps and functional imaging data. NeuroImage, 25(4), 1325-1335. https://doi.org/10.1016/j. neuroimage.2004.12.034.

Engelhard, I., van Uijen, S., \& van den Hout, M. (2010). The impact of taxing working memory on negative and positive memories. European Journal of Psychotraumatology, 1(1), 5623. https://doi. org/10.3402/ejpt.v1i0.5623.

Haier, R. J., Karama, S., Leyba, L., \& Jung, R. E. (2009). MRI assessment of cortical thickness and functional activity changes in adolescent girls following three months of practice on a visual-spatial task. BMC Research Notes, 2(174), 174. https://doi.org/10.1186/17560500-2-174.

Haier, R. J., Siegel, B. V., MacLachlan, A., Soderling, E., Lottenberg, S., \& Buchsbaum, M. S. (1992). Regional glucose metabolic changes after learning a complex visuospatial/motor task: A positron emission tomographic study. Brain Research, 570(1-2), 134-143. https://doi.org/10.1016/0006-8993(92)90573-R.

Hardwick, R. M., Rottschy, C., Miall, R. C., \& Eickhoff, S. B. (2013). A quantitative meta-analysis and review of motor learning in the human brain. NeuroImage, 67, 283-297. https://doi.org/10.1016/j. neuroimage.2012.11.020.

Holmes, E. A., Ghaderi, A., Harmer, C. J., Ramchandani, P. G., Cuijpers, P., Morrison, A. P., Roiser, J. P., Bockting, C. L. H., O'Connor, R. C., Shafran, R., Moulds, M. L., \& Craske, M. G. (2018, March 1). The lancet psychiatry commission on psychological treatments research in tomorrow's science. The Lancet Psychiatry, 5(3), 237286. https://doi.org/10.1016/S2215-0366(17)30513-8.

Holmes, E. A., \& Mathews, A. (2005). Mental imagery and emotion: A special relationship? Emotion, 5(4), 489-497. https://doi.org/10. 1037/1528-3542.5.4.489.

Holmes, E., \& Agren, T. (2020). 100 words on mental imagery - 100 words. The British Journal of Psychiatry, 216(4), 196-196. https:// doi.org/10.1192/bjp.2020.41.

Hoppe, J. M., Holmes, E. A., \& Agren, T. (2021). Exploring the neural basis of fear produced by mental imagery: Imaginal exposure in 
individuals fearful of spiders: Neural basis of fearful mental imagery. Philosophical Transactions of the Royal Society B: Biological Sciences, 376(1817). https://doi.org/10.1098/rstb.2019.0690

Horsch, A., Vial, Y., Favrod, C., Harari, M. M., Blackwell, S. E., Watson, P., Iyadurai, L., Bonsall, M. B., \& Holmes, E. A. (2017). Reducing intrusive traumatic memories after emergency caesarean section: A proof-of-principle randomized controlled study. Behaviour Research and Therapy, 94, 36-47. https://doi.org/10.1016/j.brat. 2017.03.018.

Iyadurai, L., Blackwell, S. E., Meiser-Stedman, R., Watson, P. C., Bonsall, M. B., Geddes, J. R., Nobre, A. C., \& Holmes, E. A. (2018). Preventing intrusive memories after trauma via a brief intervention involving Tetris computer game play in the emergency department: A proof-of-concept randomized controlled trial. Molecular Psychiatry, 23(3), 674-682. https://doi.org/10.1038/mp.2017.23.

James, E. L., Bonsall, M. B., Hoppitt, L., Tunbridge, E. M., Geddes, J. R., Milton, A. L., \& Holmes, E. A. (2015). Computer game play reduces intrusive memories of experimental trauma via reconsolidation-update mechanisms. Psychological Science, 26(8), 1201-1215. https://doi.org/10.1177/0956797615583071.

Kanstrup, M., Singh, L., Göransson, K. E., Widoff, J., Taylor, R. S., Gamble, B., ... \& Holmes, E. A. (2021a). Reducing intrusive memories after trauma via a brief cognitive task intervention in the hospital emergency department: an exploratory pilot randomised controlled trial. Translational psychiatry, 11(1), 1-15. https://doi.org/ 10.1038/s41398-020-01124-6.

Kanstrup, M., Kontio, E., Geranmayeh, A., Olofsdotter Lauri, K., Moulds, M. L., \& Holmes, E. A. (2021b). A single case series using visuospatial task interference to reduce the number of visual intrusive memories of trauma with refugees. Clinical Psychology \& Psychotherapy, 28(1), 109-123. https://doi.org/10.1002/cpp.2489.

Kavanagh, D. J., Freese, S., Andrade, J., \& May, J. (2001). Effects of visuospatial tasks on desensitization to emotive memories. British Journal of Clinical Psychology, 40(3), 267-280. https://doi.org/10. 1348/014466501163689.

Kavanagh, D. J., Andrade, J., \& May, J. (2005). Imaginary relish and exquisite torture: The elaborated intrusion theory of desire. Psychological Review, 112(2), 446-467. https://doi.org/10.1037/ 0033-295X.112.2.446.

Kessler, H., Holmes, E. A., Blackwell, S. E., Schmidt, A. C., Schweer, J. M., Bücker, A., Herpertz, S., Axmacher, N., \& Kehyayan, A. (2018). Reducing intrusive memories of trauma using a visuospatial interference intervention with inpatients with posttraumatic stress disorder (PTSD). Journal of Consulting and Clinical Psychology, 86(12), 1076-1090. https://doi.org/10.1037/ccp0000340.

Lang, P. J. (1979). A bio-informational theory of emotional imagery. Psychophysiology, 16(6), 495-512. https://doi.org/10.1111/j.14698986.1979.tb01511.x.

Lau-Zhu, A., Holmes, E. A., Butterfield, S., \& Holmes, J. (2017). Selective association between Tetris game play and visuospatial working memory: A preliminary investigation. Applied Cognitive Psychology, 31(4), 438-445. https://doi.org/10.1002/acp.3339.

Munzert, J., Lorey, B., \& Zentgraf, K. (2009). Cognitive motor processes: The role of motor imagery in the study of motor representations. Brain Research Reviews, 60(2), 306-326. https://doi.org/10.1016/j. brainresrev.2008.12.024.

Pearson, J. (2019). The human imagination: The cognitive neuroscience of visual mental imagery. Nature Reviews Neuroscience, 20(10), 624-634. https://doi.org/10.1038/s41583-019-0202-9.

Price, R. B., Paul, B., Schneider, W., \& Siegle, G. J. (2013). Neural correlates of three neurocognitive intervention strategies: A preliminary step towards personalized treatment for psychological disorders. Cognitive Therapy and Research, 37(4), 657-672. https://doi. org/10.1007/s10608-012-9508-x.

Rietschel, J. C., Miller, M. W., Gentili, R. J., Goodman, R. N., Mcdonald, C. G., \& Hatfield, B. D. (2012). Cerebral-cortical networking and activation increase as a function of cognitive-motor task difficulty. Biological Psychology, 90(2), 127-133. https://doi.org/10.1016/j. biopsycho.2012.02.022.

Sheth, B. R., \& Young, R. (2016). Two visual pathways in primates based on sampling of space: exploitation and exploration of visual information. Frontiers in neuroscience, 10, 37. https://doi.org/10.3389/ fnint.2016.00037.

Singh, L., Espinosa, L., Ji, J. L., Moulds, M. L., \& Holmes, E. A. (2020). Developing thinking around mental health science: The example of intrusive, emotional mental imagery after psychological trauma. Cognitive Neuropsychiatry, 25(5), 348-363. https://doi.org/10. 1080/13546805.2020.1804845.

Skorka-Brown, J., Andrade, J., \& May, J. (2014). Playing ‘Tetris' reduces the strength, frequency and vividness of naturally occurring cravings. Appetite, 76, 161-165. https://doi.org/10.1016/j.appet. 2014.01.073.

Skorka-Brown, J., Andrade, J., Whalley, B., \& May, J. (2015). Playing Tetris decreases drug and other cravings in real world settings. Addictive Behaviors, 51, 165-170. https://doi.org/10.1016/j. addbeh.2015.07.020.

Tzourio-Mazoyer, N., Landeau, B., Papathanassiou, D., Crivello, F., Etard, O., Delcroix, N., Mazoyer, B., \& Joliot, M. (2002). Automated anatomical labeling of activations in SPM using a macroscopic anatomical parcellation of the MNI MRI single-subject brain. NeuroImage, 15(1), 273-289. https://doi.org/10.1006/nimg. 2001.0978

Weiner, K. S., Natu, V. S., \& Grill-Spector, K. (2018). On object selectivity and the anatomy of the human fusiform gyrus. Neuroimage, 173, 604-609. https://doi.org/10.1016/j.neuroimage.2018.02.040.

Publisher's Note Springer Nature remains neutral with regard to jurisdictional claims in published maps and institutional affiliations. 